\title{
Optogenetic Activation of Excitatory Premotor Interneurons Is Sufficient to Generate Coordinated Locomotor Activity in Larval Zebrafish
}

\author{
Emma Eklöf Ljunggren, ${ }^{\star}$ Sabine Haupt, ${ }^{\star}$ Jessica Ausborn, Konstantinos Ampatzis, and Abdeljabbar El Manira \\ Department of Neuroscience, Karolinska Institute, 17177 Stockholm, Sweden
}

Neural networks in the spinal cord can generate locomotion in the absence of rhythmic input from higher brain structures or sensory feedback because they contain an intrinsic source of excitation. However, the molecular identity of the spinal interneurons underlying the excitatory drive within the locomotor circuit has remained unclear. Using optogenetics, we show that activation of a molecularly defined class of ipsilateral premotor interneurons elicits locomotion. These interneurons represent the excitatory module of the locomotor networks and are sufficient to produce a coordinated swimming pattern in zebrafish. They correspond to the V2a interneuron class and express the transcription factor Chx10. They produce sufficient excitatory drive within the spinal networks to generate coordinated locomotor activity. Therefore, our results define the V2a interneurons as the excitatory module within the spinal locomotor networks that is sufficient to initiate and maintain locomotor activity.

\section{Introduction}

The richness of the motor repertoire produced by vertebrates arises from a variety of neural circuits each underlying a specific motor function (Shepherd and Grillner, 2010). Defining the neuronal components of these circuits and assigning them a functional role has remained a major challenge. Spinal locomotor circuits, with their measurable outputs, afford linking the activity of a class of neurons to a global motor pattern. They possess the inherent ability to generate coordinated activity because they comprise excitatory and inhibitory interneuron modules (Grillner, 2003; El Manira and Grillner, 2008; Goulding, 2009; Grillner and Jessell, 2009; Fetcho and McLean, 2010; Sillar, 2010; Büschges et al., 2011). The prevailing view is that the excitatory tone underlying locomotion arises from ipsilateral excitatory interneurons within the locomotor circuits. However, the molecular identity of this excitatory module has remained unclear.

In lamprey, Xenopus tadpoles, and larval zebrafish, ipsilateral excitatory interneurons have been shown to activate motoneurons monosynaptically (Grillner, 2003; Kimura et al., 2006; Roberts et al., 2008, 2010). These interneurons are homologous to the V2a interneurons that express the transcription factor Chx10 in zebrafish and mammals (Kimura et al., 2006). Moreover, in larval zebrafish, ablation of a proportion of the $\mathrm{V} 2 \mathrm{a}$ interneurons decreases

Received Sept. 24, 2013; revised Nov. 6, 2013; accepted Nov. 11, 2013.

Author contributions: E.E.L. and A.E.M. designed research; S.H., J.A., and K.A. performed research; E.E.L., S.H., J.A., K.A., and A.E.M. analyzed data; E.E.L., J.A., and A.E.M. wrote the paper.

This work was funded by a grant from the Swedish Research Council and Karolinska Institute. We thank S. Grillner and C. Wyart for comments and critical discussion of this manuscript, H. Baier for providing the fish lines used in this study, and S.-I. Higashijima for providing the Chx10 antibody.

*E.E.L. and S.H. contributed equally to this work.

Correspondence should be addressed to Abdel El Manira, Department of Neuroscience, Karolinska Institute, 171 77 Stockholm, Sweden. E-mail: Abdel.EIManira@ki.se.

DOI:10.1523/JNEUROSCI.4087-13.2014

Copyright $\odot 2014$ the authors $\quad 0270-6474 / 14 / 340134-06 \$ 15.00 / 0$ the excitability of the spinal circuits, suggesting that these interneurons are necessary for the normal expression of the locomotor rhythm (Eklöf Ljunggren et al., 2012). However, the sufficiency of these interneurons for the generation of a coordinated locomotor rhythm has not yet been clarified.

In this study, we sought to assess the sufficiency of the V2a interneuron class for the generation of locomotion. To this end, we used optogenetic tools in transgenic zebrafish (Scott et al., 2007) that selectively express channelrhodopsin-2 (ChR2) in the V2a interneurons. We show that the V2a interneurons form an excitatory network sufficient to generate a coordinated locomotor pattern. Selective optogenetic stimulation of these interneurons produces synaptic excitation in other interneurons of the same class. The excitatory drive generated by V2a interneurons is sufficient to produce swimming activity that is characterized by the typical left-right and rostrocaudal coordination. These results identify the V2a interneurons as the excitatory module within the spinal circuit that provides the sufficient excitatory tone to initiate and maintain a coordinated locomotor rhythm.

\section{Materials and Methods}

Zebrafish lines and care. All experimental protocols were approved by the animal research committee, Stockholm. Four transgenic zebrafish lines were used: the Gal4 ${ }^{\text {s1011t }}$ (Et(-1.5hsp70I:Gal4-VP16)s1011t), in which Gal4-VP16 was expressed in circumferential ipsilateral descending (CiD)-like interneurons (Scott et al., 2007), and the UAS:Kaede ${ }^{\text {s1999t }}$ [Tg(UAS-E1b:Kaede)s1999t; Davison et al., 2007], UAS:GFP, and UAS: ChR2(H134R)-mCherry lines [Tg(UAS:ChR2(H134R)-mCherry) s1986t; Schoonheim et al., 2010], which carry Kaede, GFP, and ChR2(H134R)-mCherry, respectively, under an upstream activated sequence (UAS) that allows expression only when Gal4 is present. Experiments were performed on 4- to 5-d-old larval zebrafish of either sex at room temperature $\left(\sim 22^{\circ} \mathrm{C}\right)$.

Backfilling and imaging of motoneurons and long projecting interneurons. Larval zebrafish were anesthetized in $0.03 \%$ tricane methanesulfo- 
nate (MS222; Sigma-Aldrich) and then transferred to extracellular solution containing the following (in mM): $134 \mathrm{NaCl}, 2.9 \mathrm{KCl}, 1.2 \mathrm{MgCl}_{2}$, $2.1 \mathrm{CaCl}_{2}, 10$ HEPES, 10 glucose, pH 7.8, osmolarity 290 mOsm. Motoneurons were backfilled by applying a small amount of tetramethylrhodamine dextran (3000 MW; Invitrogen) into the axial muscles using sharp tungsten pins. Descending interneurons were backfilled by dye injections into the caudal part of the spinal cord using a glass capillary needle filled with the fluorescent tracer diluted in extracellular solution. After injections, the animals were left to recover for $1 \mathrm{~h}$ to allow retrograde transport of the dye. The larvae were then fixed in $4 \%$ paraformaldehyde (PFA) in phosphate buffer (PBS, $0.01 \mathrm{M}, \mathrm{pH} 7.4$ ) for $3 \mathrm{~h}$ at room temperature before imaging neurons using a laser scanning confocal microscope (LSM 510 Meta; Zeiss).

Immunohistochemistry. Larval zebrafish were fixed in $4 \%$ PFA for $3 \mathrm{~h}$ at room temperature, washed in PBS containing 0.1\% Triton X-100 $(2 \times 5$ min; Sigma), and blocked with 1\% Western Blocking Solution (Roche) for $30 \mathrm{~min}$ before incubation with the primary antibody (guinea pig anti-Chx10, 1:500 in 1\% Western Blocking Solution; Kimura et al., 2008) overnight at $4^{\circ} \mathrm{C}$. After washing in PBS, larvae were incubated with the secondary antibody (anti-gp-Cy3, 1:200, in PBS/Triton X-100) overnight at $4^{\circ} \mathrm{C}$, washed $6 \times 20$ min with PBS, and mounted on gelatinized glass slides in $75 \%$ glycerol solution (in PBS) before imaging.

Electrophysiology. The experimental procedure for electrophysiological experiments was performed as described previously (Gabriel et al., 2008, 2009; Eklöf Ljunggren et al., 2012). The animals were spinalized within the first five segments of the spinal cord and swimming activity was elicited by using light stimulation (blue light; $465 \mathrm{~nm}$ ) delivered by a custom-made LED system (ACULED VHL; PerkinElmer). The intensity of the stimulation was gradually increased until fictive swimming was elicited reliably at every trial. For whole-cell patch-clamp recordings, fish were treated in the same way as for extracellular recordings, except they were placed on the side and muscles were removed over one segment. Patch-clamp electrodes were pulled from borosilicate glass $(1.5 \mathrm{~mm}$ outer diameter, $0.87 \mathrm{~mm}$ inner diameter; Hilgenberg) on a vertical puller (Narishige) and filled with intracellular solution containing the following (in $\mathrm{mM}$ ): 120 potassium gluconate, $5 \mathrm{KCl}, 10 \mathrm{HEPES}, 4 \mathrm{ATP}-\mathrm{Mg}^{2+}$, $0.3 \mathrm{GTP}-\mathrm{Na}^{+}, 10 \mathrm{Na}^{+}$-phosphocreatine, $\mathrm{pH}$ 7.4, with $\mathrm{KOH}, 275 \mathrm{mOsm}$, yielding resistances of $8-12 \mathrm{M} \Omega$.

Data acquisition and analysis. Data analysis was performed using cross- or auto-correlation analysis in Spike2 (Cambridge Electronic Design). Period and phase values of the motor pattern were calculated from cross- and auto-correlations as described previously (Eklöf Ljunggren et al., 2012).

\section{Results}

\section{Expression of Gal4 in V2a interneurons}

To determine whether activating V2a interneurons can induce locomotor activity, we used the zebrafish Gal4 transgenic line Gal4 ${ }^{\text {s1011t }}$. This line has been obtained from an enhancer trap screen (Scott et al., 2007) and drives the expression of Channelrhodopsin-2(H134R;ChR2) when crossed with a UAS: ChR2(H134R)-mCherry line (Arrenberg et al., 2009; Baier and Scott, 2009; Wyart et al., 2009; Del Bene and Wyart, 2012). We first investigated whether the Gal4 expression was selective to V2a interneurons by crossing the Gal4 ${ }^{\text {s1011t }}$ with UAS:GFP or UAS: Kaede. We determined the morphology and molecular identity of the interneurons in 4- to 5-d-old larvae. The labeled interneurons had a primary axon that was extending ventrally, after which it turned and projected caudally on the ipsilateral side of the spinal cord (Fig. 1A). To rule out the possibility of Gal4 expression in motoneurons, we retrogradely labeled motoneurons in the double-transgenic Gal4 ${ }^{\text {s1011t }}$,UAS:Kaede by injecting a fluorescent tracer into the axial musculature. We did not observe colocalization of rhodamine and Kaede in motoneurons in any of these experiments $(n=8$; Fig. $1 B)$. To determine whether the Gal4-expressing interneurons had long descending axons, we injected Rhodamine Dextran into the caudal part of the spinal cord
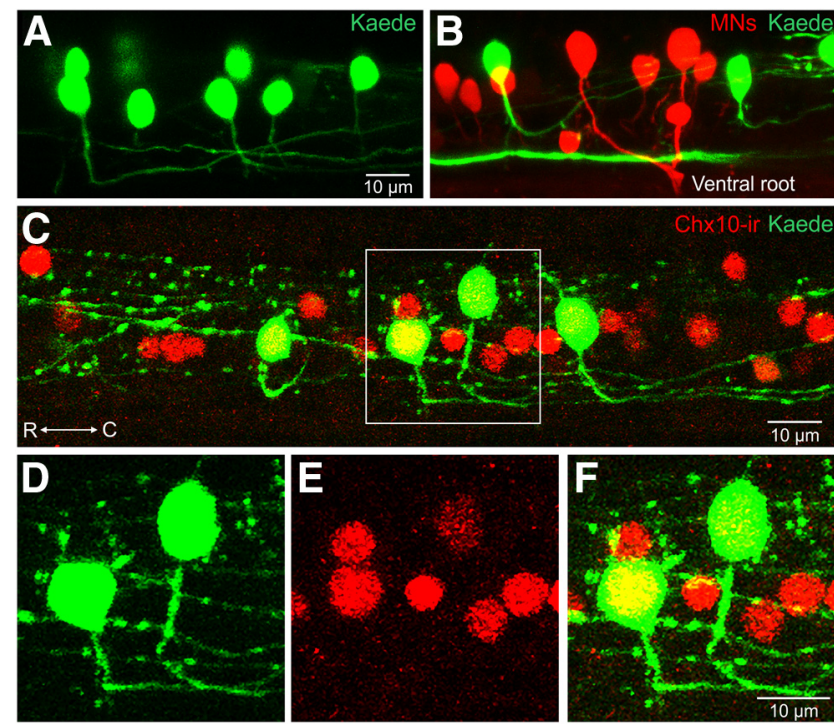

Figure 1. Specific expression of Gal4 in V2a interneurons. $\boldsymbol{A}$, Interneurons expressing Gal4 display the characteristic morphological features of V2a interneurons. $\boldsymbol{B}$, Expression of Gal4 is not detected in motoneurons. $\boldsymbol{C}$, Interneurons expressing Gal4 also express the transcription factor Chx10. Confocal reconstruction of a part of the spinal cord in the mid-body region of a double-transgenic larvae zebrafish, Gal4 ${ }^{\text {s1011t }}$;UAS:Kaede, in which immunohistochemistry with a Chx10 antibody was performed. D, Enlarged picture of the white box in C showing two V2a interneurons expressing Kaede. $\boldsymbol{E}$, Same area as in $\boldsymbol{D}$ showing neurons immunoreactive against Chx10. $\boldsymbol{F}$, Overlay of $\boldsymbol{D}$ and $\boldsymbol{E}$ showing that V2a interneurons were double labeled for Kaede and $\mathrm{Chx} 10$.

while imaging rostral interneurons in the double transgenics Gal4 ${ }^{\text {s1011t }}$;UAS:Kaede or Gal4 ${ }^{\text {s1011t }}$;UAS:GFP. This retrograde labeling revealed interneurons that were double labeled with rhodamine and either GFP or Kaede, indicating that the labeled interneurons projected over several segments caudally in the spinal cord (data not shown). The morphological features of these interneurons (with an ipsilateral and descending axon) resembled those of V2a interneurons, also called CiD neurons (Hale et al., 2001). To further confirm that the interneurons expressing Gal4 correspond to V2a interneurons, we performed immunohistochemical experiments using an antibody against the transcription factor Chx10, which is selectively expressed in these interneurons (Kimura et al., 2006). In all experiments $(n=6$ larvae), all interneurons expressing GFP or Kaede were also immunoreactive for Chx10 (Fig. $1 C-F$ ) and represented $21.3 \pm$ $3.1 \%$ of Chx10-positive neurons. Together, these results indicate that the Gal4 ${ }^{\text {s1011t }}$ zebrafish line is selective for V2a interneurons in the spinal cord.

\section{Light activation of V2a interneurons induces swimming activity}

To determine whether activation of V2a interneurons is sufficient to generate swimming, we first assessed whether the target interneurons can be selectively activated using optogenetics in the double-transgenic Gal4 ${ }^{\text {s1011t }}$;UAS:ChR2(H134R)-mCherry larvae. Whole-cell patch-clamp recordings were made from neurons expressing ChR2 identified by the expression of mCherry. Application of a short pulse $(10 \mathrm{~ms})$ of blue light $(465 \mathrm{~nm})$ with graded light power depolarized the membrane potential of the recorded V2a interneurons gradually until firing of action potentials could be reliably induced ( $n=21$; Fig. $2 A$ ). In addition to producing a direct depolarization of the recorded V2a interneurons, activation of ChR2 with a short light pulse $(10 \mathrm{~ms})$ elicited 


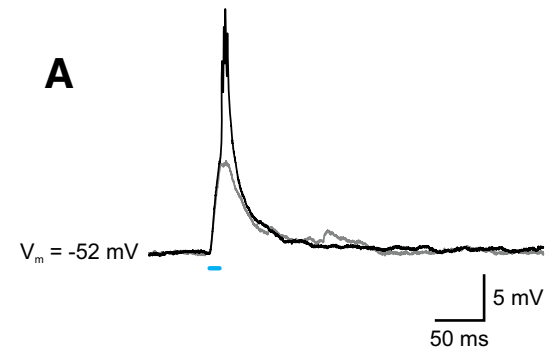

B

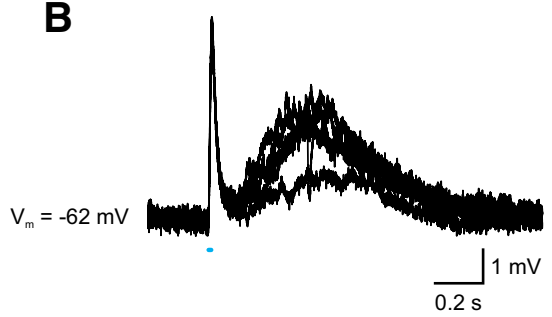

C

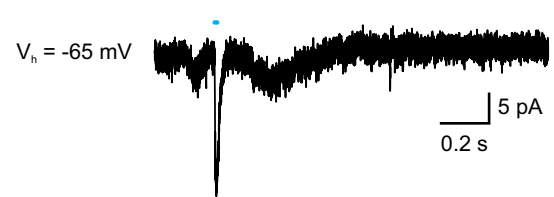

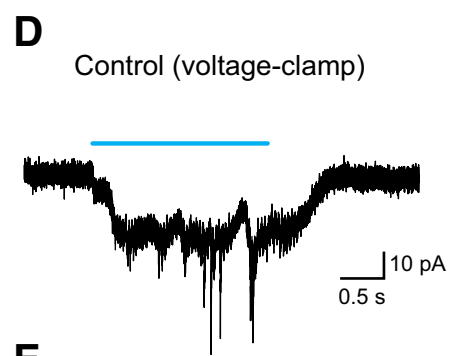

E

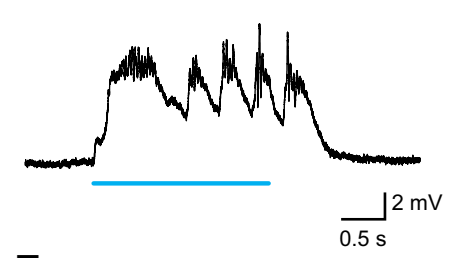

$\mathbf{F}$

AP-5 and GYKI

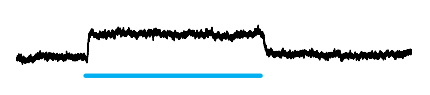

G

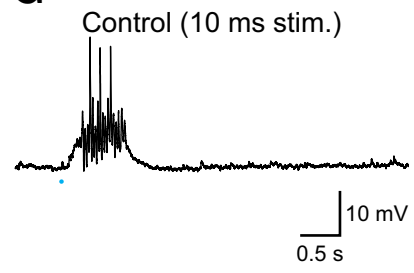

H

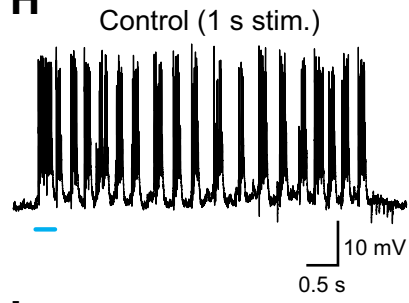

AP-5 and GYKI

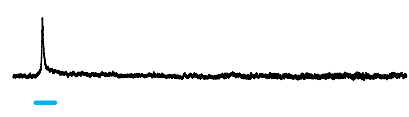

Figure 2. Optogenetic activation of interconnected V2a interneurons elicits rhythmic burst activity. $\boldsymbol{A}$, Optical stimulation of a V2a interneuron expressing ChR2 induced a membrane potential depolarization that could reach the threshold for firing action potentials. B, Optogenetic activation of V2a interneurons induced a ChR2-mediated membrane potential depolarization followed by a delayed and slower excitation in current-clamp conditions. C, In voltage-clamp conditions, the same stimulation induced a ChR2-mediated inward current followed by a synaptic inward excitatory current. $\boldsymbol{D}$, In voltage-clamp conditions, long-lasting optical stimulation induced a tonic inward current associated with episodes of synaptic currents in a V2a interneuron. $\boldsymbol{E}$, The same interneuron recorded in current-clamp showed an episode of rhythmic synaptic oscillations superimposed on a tonic depolarization. $F$, Blocking ionotropic glutamate receptors abolished the synaptic oscillations, leaving only the tonic ChR2-mediated depolarization. $\boldsymbol{G}$, A short optical stimulation induced a short bursting activity in the recorded V2a interneuron. $\boldsymbol{H}$, Increasing the duration of the stimulation resulted in a long bout of bursting activity that outlasted the stimulus. I, Rhythmic bursting was abolished after blocking ionotropic receptors and only the direct ChR2-induced depolarization persisted.

slow excitatory synaptic responses in some V2a interneurons $(n=6$; Fig. $2 B)$. These responses could be seen clearly as inward currents in the same V2a interneurons recorded in voltage-clamp conditions (Fig. 2C).

We also investigated whether activation of the V2a interneuron population was able to induce rhythmic activity in these interneurons. In voltage-clamp conditions, application of a long (2 s) light pulse induced a tonic inward current in the V2a interneurons that was associated with rhythmic synaptic currents (Fig. $2 D)$. Conversely, in current-clamp from the same V2a interneurons, optical stimulation induced rhythmic membrane potential oscillations superimposed on a ChR2-induced tonic depolarization (intra-episode burst frequency: $25.0 \pm 1.4 \mathrm{~Hz} ; n=6$; Fig. $2 E)$. The synaptic activity and the associated rhythmic membrane potential oscillations were completely abolished by blocking NMDA and AMPA receptors (Fig. $2 F$ ). The duration and intensity of the rhythmic activity induced in V2a interneurons varied with the duration of the optical stimulation. In some preparations, light stimulation as short as $10 \mathrm{~ms}$ triggered a short bout of rhythmic membrane potential oscillations in V2a interneurons $(n=4$, Fig. $2 G)$. Increasing the stimulus duration to $1 \mathrm{~s}$ resulted in a long swimming bout with episodes of rhythmic bursting typical of the beat-and-glide larval swimming pattern (Fig. $2 \mathrm{H}$ ). This rhythmic bursting was completely inhibited by the NMDA and AMPA receptor antagonists AP5 $(50 \mu \mathrm{M})$ and GYKI 52466 $(30 \mu \mathrm{M})$, respectively ( $n=5$; Fig. $2 I$ ). Therefore, the ChR2induced direct membrane potential depolarization of cells in the
Gal4 s1011t;UAS:ChR(H134R)-mCherry line was not sufficient to elicit rhythmic bursting. These results indicate that V2a interneurons form an excitatory network that is sufficient to provide the necessary excitation underlying the rhythm generation in the spinal locomotor circuit.

Finally, we investigated whether the burst activity recorded in single V2a interneurons corresponds to swimming activity. We recorded peripheral nerve activity in spinalized and immobilized larval zebrafish (Fig. 3A). Optical stimulation of a long duration induced a swimming episode characterized by alternation of activity between the left and right body sides and a rostrocaudal propagation of activity $(n=10$; Fig. $3 B, C)$. The burst frequency ranged between 18 and $25 \mathrm{~Hz}$, which corresponds to normal swimming behavior in intact larval zebrafish (Buss and Drapeau, 2001; Masino and Fetcho, 2005). Swimming was elicited when threshold intensity was reached. In addition, once swimming activity was induced, the burst frequency remained constant at $23.4 \pm 0.9 \mathrm{~Hz}(n=10)$. These results show that swimming activity indeed emerges from the activity of an underlying V2a interneuron network and that the excitatory drive provided by these V2a interneurons is sufficient to initiate the swimming motor pattern.

Rhythmic activity induced by stimulation of V2a interneurons does not require inhibition

A previous study showed that optical stimulation of a class of GABAergic neurons, Kolmer-Agduhr (KA) cells, induced swim- 
A

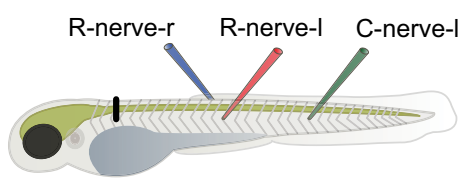

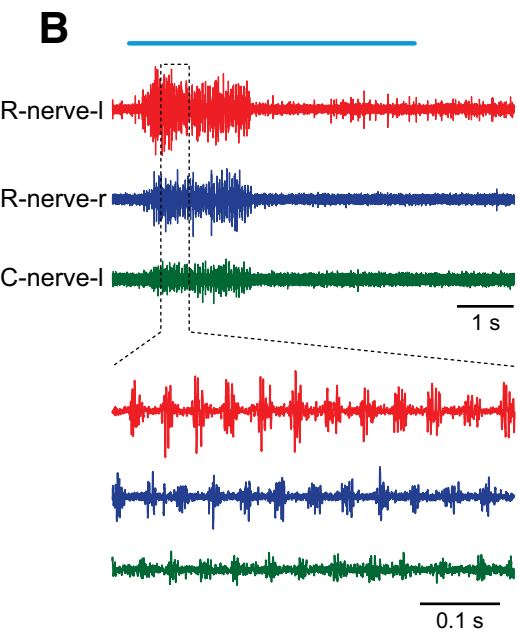

C

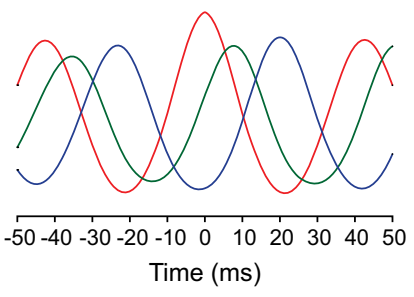

D

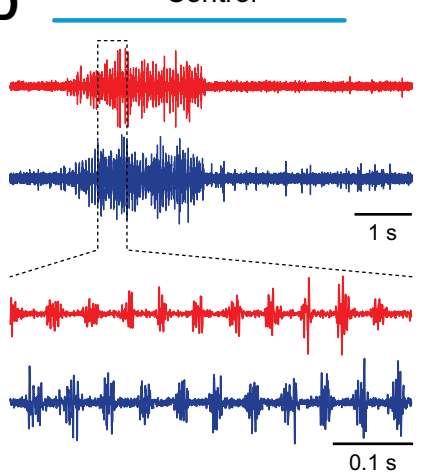

E

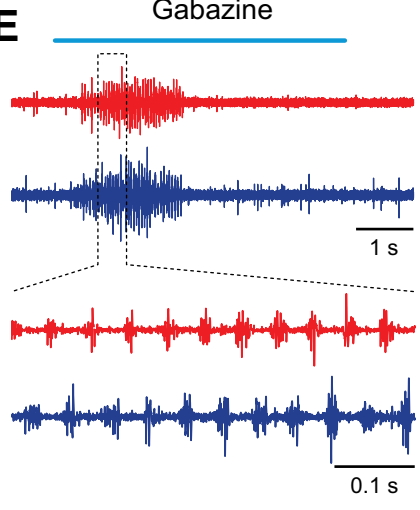

F

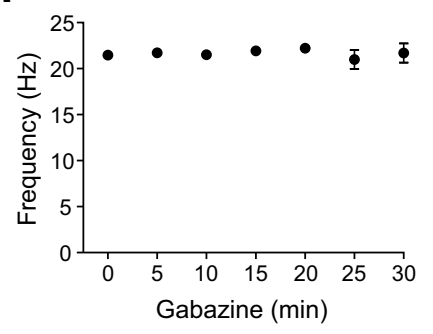

G

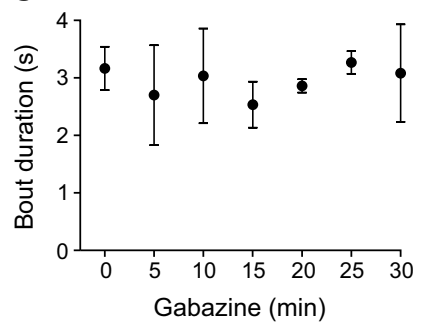

Figure 3. Optogenetic activation of V2a interneurons generates coordinated locomotor activity. $\boldsymbol{A}$, Experimental setup with a spinalized larval zebrafish in which motor activity is monitored by recording motor nerves on the left (R-nerve-I) and right (R-nerve-r) side of the same rostral segment and on the left side of a caudal segment (C-nerve-I). $\boldsymbol{B}, \mathbf{O p t i c a l}$ stimulation induced a bout of locomotor activity with the characteristic left-right alternation and rostrocaudal delay. $C$, Auto-and cross-correlation analysis showing the coordinated pattern of the swimming activity induced by V2a interneuron activation. $\boldsymbol{D}, \boldsymbol{E}$, Swimming activity induced by optical stimulation of V2a interneurons was not affected by blocking $\mathrm{GABA}_{\mathrm{A}}$ receptors with gabazine. $\boldsymbol{F}$, Gabazine did not affect the frequency of the swimming activity. $\mathbf{G}$, Bout duration of the swimming activity was not affected by gabazine.

ming activity that was dependent on activation of $\mathrm{GABA}_{\mathrm{A}}$ receptors (Wyart et al., 2009). To determine whether optogenetically induced swimming activity was dependent on $\mathrm{GABA}_{\mathrm{A}}$ receptor activation, the specific antagonist gabazine (30 $\mu \mathrm{M} ; n=5)$ was used. Gabazine had no effect on either left-right coordination of the swimming activity (Fig. $3 D, E$ ) or on the light threshold for inducing swimming. Once the swimming activity was induced, the burst frequency and the bout duration remained constant throughout the entire time of gabazine application (Fig. $3 F, G$ ). These results indicate that $\mathrm{GABA}_{\mathrm{A}}$ receptor activation is not necessary for the expression or regulation of the swimming activity induced by optical stimulation of the glutamatergic V2a interneurons.

Another important inhibitory component of the spinal locomotor circuits is the reciprocal inhibition ensuring left-right alternation. Indeed, locomotor activity is thought to be the result of ipsilateral glutamatergic excitatory drive and crossed glycinergic inhibition, which ensures left-right alternation. If activation of V2a interneurons is sufficient to produce the basic rhythmicity within the spinal locomotor network, this should be possible even in the absence of inhibition. To test this, glycinergic synaptic transmission was blocked with strychnine. In controls, lightactivation of $\mathrm{V} 2 \mathrm{a}$ interneurons induced a bout of coordinated swimming activity (Fig. 4A, B). Blocking of glycine receptors with strychnine ( $1 \mu \mathrm{M}, n=6)$ transformed the alternating activity into synchronous bursting of left and right motor nerves (Fig. 4C,D). Activation of V2a interneurons with long light pulses induced episodic bursting that always occurred simultaneously in left and right motor nerves (Fig. $4 E, F$ ). This indicates that the excitatory drive provided by $\mathrm{V} 2 \mathrm{a}$ interneurons is sufficient to produce slow episodic motor activity in the absence of crossed inhibitory connections.

\section{Discussion}

The expression of the locomotor rhythm requires an intrinsic excitatory drive from interneurons within the locomotor circuits (Grillner, 2003; Roberts et al., 2008; Grillner and Jessell, 2009). However, the molecular identity of the interneurons sufficient to initiate the rhythmic activity of these circuits is still unclear. We used optogenetics to selectively activate a molecularly defined class of interneurons. Our results show that light activation of V2a interneurons selectively expressing ChR2 is sufficient to generate locomotor activity in zebrafish. The selective expression of ChR2 in V2a interneurons was determined immunohistochemically, morphologically, and electrophysiologically. Light stimulation induced a ChR2-induced current, resulting in activation of these interneurons followed by synaptic responses arising from activation of neighboring interneurons. Optogenetic stimulation 
A

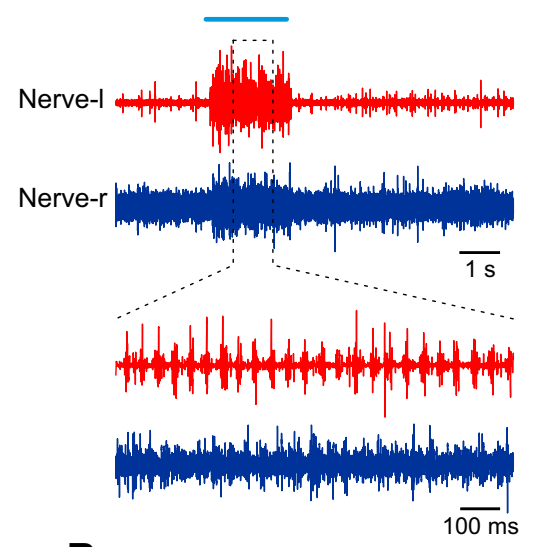

B

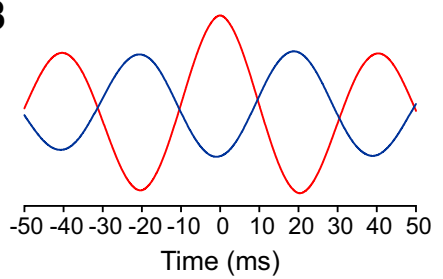

C Strychnine

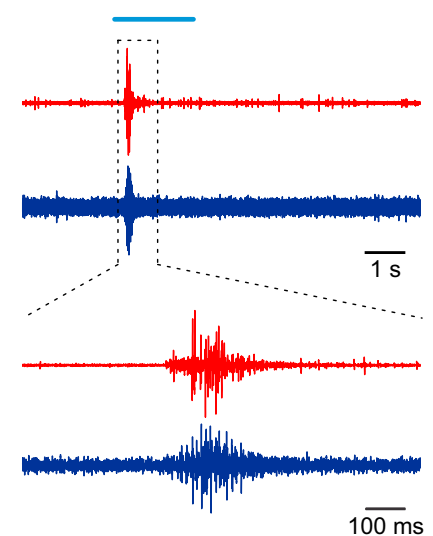

D

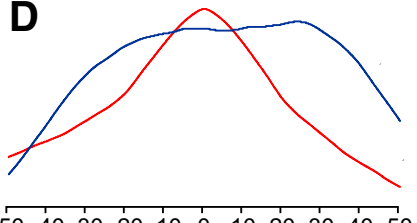

$-50-40-30-20-10 \quad 0 \quad 10 \quad 20 \quad 30 \quad 4050$ Time (ms)
E Strychnine

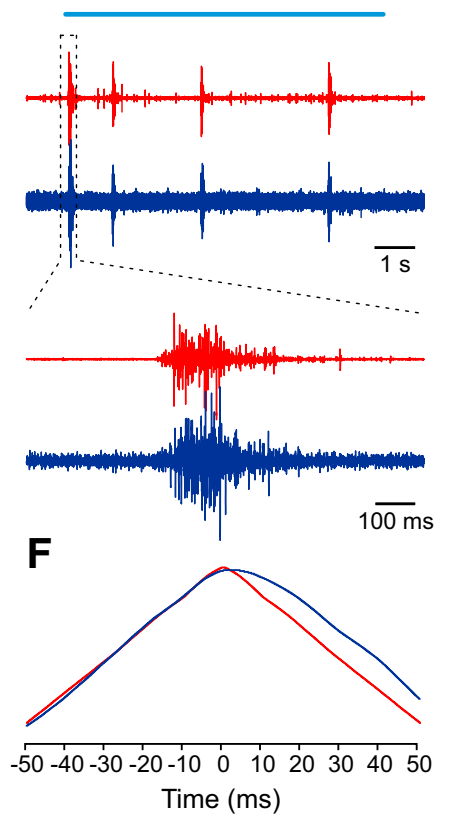

Figure 4. Optogenetic activation produces repetitive bursting in the absence of inhibition. $\boldsymbol{A}, \boldsymbol{B}$, Recording of motor nerves on the left (Nerve-I) and right (Nerve-r) side of the same segment. Optical stimulation of $\mathrm{V} 2 \mathrm{a}$ interneurons elicited a swimming bout in a spinalized animal displaying left-right alternation. $\boldsymbol{C}-\boldsymbol{F}$, Left-right alternation was abolished after blocking glycinergic commissural synaptic transmission with strychnine. $E, F$, Long-lasting optical stimulation of V2a interneurons produced rhythmic burst activity that occurred synchronously in left and right motor nerves.

of V2a interneurons in spinalized preparations was sufficient to induce rhythmic swimming activity characterized by left-right alternation and a rostrocaudal delay. This activity was mediated by synaptic activation of ionotropic glutamate receptors. Blocking of $\mathrm{GABA}_{\mathrm{A}}$ receptors did not affect the swimming activity, which was transformed into synchronous slow, rhythmic bursting by blocking crossed glycinergic inhibition. Therefore, these results indicate that $\mathrm{V} 2 \mathrm{a}$ interneurons represent the intrinsic excitatory interneuron module within the spinal networks that is sufficient to generate the basic rhythm underlying locomotion.

Assigning function to specific classes of interneurons within the spinal locomotor circuits is an important step toward building a function-based connectivity scheme. We previously showed that acute ablation of V2a interneurons in zebrafish decreases the excitability of the locomotor network and reduces the swimming burst frequency (Roberts et al., 2008; Eklöf Ljunggren et al., 2012), suggesting that they contribute to the excitatory drive necessary for the normal expression of locomotion. Our results show that V2a interneurons are not just necessary but also sufficient to drive the activity of the whole locomotor circuit and hence produce coordinated activity. Homologous interneuron classes are found in other vertebrates, including lamprey, Xenopus tadpoles, and mice (Kimura et al., 2006, 2008; Dasen and Jessell, 2009; Goulding, 2009; Grillner and Jessell, 2009; Fetcho and McLean, 2010). Based on their connectivity with motoneurons and other interneurons delineated in lampreys and Xenopus tadpoles, the excitatory interneurons have been suggested to play an important role during locomotion (Grillner, 2003; Roberts et al., 2008). In these preparations, however, it has not been possible to define the molecular profile of these interneurons and selectively manipulate their activity as a population. The use of optogenetic tools has enabled us to show directly that activation of V2a interneurons alone is sufficient to contribute the necessary excitatory burst activity underlying locomotion. The incremental recruitment of these interneurons can provide increasing excitatory drive to regulate the swimming frequency (El Manira and Grillner, 2008; McLean et al., 2008; Fetcho and McLean, 2010; Gabriel et al., 2011; Ausborn et al., 2012).

The induced swimming activity relies on the activation of ionotropic glutamate receptors and does not depend on the activation of $\mathrm{GABA}_{\mathrm{A}}$ receptors. A previous study has shown that optical activation of the GABAergic KA cells surrounding the central canal was able to produce swimming activity in larval zebrafish, which could be blocked by a $\mathrm{GABA}_{\mathrm{A}}$ receptor antagonist (Wyart et al., 2009). At this early developmental stage, the chloride reversal potential may still be more depolarized and $\mathrm{GABA}_{\mathrm{A}}$ activation could lead to depolarization of other neurons such as V2a interneurons that in turn initiate swimming. Another possibility is that V2a interneurons are inhibited by GABA released from KA cells and that V2a interneurons in addition display rebound firing properties that allow them to trigger the activity of the spinal network. It is thus possible that V2a interneurons are the downstream interneurons producing swimming activity after activation of KA cells. Further analysis is, however, required to determine how KA cells and V2a interneurons interact and whether they act on the same or separate neuronal targets to induce swimming.

Several studies have attempted to define the excitatory interneurons within the locomotor network. Lamprey lesion studies have shown that a basic rhythmic activity is mediated by a network of excitatory interneurons (Cangiano and Grillner, 2003, 2005). Descending excitatory neurons in the brainstem have been shown to make reciprocal synapses with each other in Xenopus tadpole (Li et al., 2006; Li, 2011) and optical activation of these neurons in zebrafish is able to elicit locomotion (Kimura et al., 2013). Recently, the importance of glutamatergic neurons in inducing locomotor rhythm was confirmed in the mouse spinal cord (Hägglund et al., 2010). However, none of these studies was 
able to identify the molecular signature of the spinal excitatory interneuron module generating the locomotor rhythm. Using optogenetic tools, we now show that V2a interneurons expressing Chx10 represent the excitatory unit generating the basic rhythm underlying locomotion. Given the homology of these interneurons across several vertebrate species, it is possible that their function is conserved during evolution. Therefore, the identification of V2a interneurons as necessary and sufficient for initiating locomotor activity contributes importantly to our understanding of the neuronal processing underlying motor behavior.

\section{References}

Arrenberg AB, Del Bene F, Baier H (2009) Optical control of zebrafish behavior with halorhodopsin. Proc Natl Acad Sci U S A 106:17968-17973. CrossRef Medline

Ausborn J, Mahmood R, El Manira A (2012) Decoding the rules of recruitment of excitatory interneurons in the adult zebrafish locomotor network. Proc Natl Acad Sci U S A 109:E3631-3639. CrossRef Medline

Baier H, Scott EK (2009) Genetic and optical targeting of neural circuits and behavior-zebrafish in the spotlight. Curr Opin Neurobiol 19:553-560. CrossRef Medline

Büschges A, Scholz H, El Manira A (2011) New moves in motor control. Curr Biol 21:R513-R524. CrossRef Medline

Buss RR, Drapeau P (2001) Synaptic drive to motoneurons during fictive swimming in the developing zebrafish. J Neurophysiol 86:197-210. Medline

Cangiano L, Grillner S (2003) Fast and slow locomotor burst generation in the hemispinal cord of the lamprey. J Neurophysiol 89:2931-2942. CrossRef Medline

Cangiano L, Grillner S (2005) Mechanisms of rhythm generation in a spinal locomotor network deprived of crossed connections: the lamprey hemicord. J Neurosci 25:923-935. CrossRef Medline

Dasen JS, Jessell TM (2009) Hox networks and the origins of motor neuron diversity. Curr Top Dev Biol 88:169-200. CrossRef Medline

Davison JM, Akitake CM, Goll MG, Rhee JM, Gosse N, Baier H, Halpern ME, Leach SD, Parsons MJ (2007) Transactivation from Gal4-VP16 transgenic insertions for tissue-specific cell labeling and ablation in zebrafish. Dev Biol 304:811-824. CrossRef Medline

Del Bene F, Wyart C (2012) Optogenetics: a new enlightenment age for zebrafish neurobiology. Developmental neurobiology 72:404414. CrossRef Medline

Eklöf Ljunggren E, Haupt S, Ausborn J, Dehnisch I, Uhlén P, Higashijima SI, El Manira A (2012) Origin of excitation underlying locomotion in the spinal circuit of zebrafish. Proc Natl Acad Sci U S A

El Manira A, Grillner S (2008) Switching gears in the spinal cord. Nat Neurosci 11:1367-1368. CrossRef Medline

Fetcho JR, McLean DL (2010) Some principles of organization of spinal neurons underlying locomotion in zebrafish and their implications. Ann N Y Acad Sci 1198:94-104. CrossRef Medline

Gabriel JP, Mahmood R, Walter AM, Kyriakatos A, Hauptmann G, Calabrese RL, El Manira A (2008) Locomotor pattern in the adult zebrafish spinal cord in vitro. J Neurophysiol 99:37-48. Medline

Gabriel JP, Mahmood R, Kyriakatos A, Söll I, Hauptmann G, Calabrese RL, El Manira A (2009) Serotonergic modulation of locomotion in zebrafish: endogenous release and synaptic mechanisms. J Neurosci 29:1038710395. CrossRef Medline

Gabriel JP, Ausborn J, Ampatzis K, Mahmood R, Eklöf-Ljunggren E, El
Manira A (2011) Principles governing recruitment of motoneurons during swimming in zebrafish. Nat Neurosci 14:93-99. CrossRef Medline Goulding M (2009) Circuits controlling vertebrate locomotion: moving in a new direction. Nat Rev Neurosci 10:507-518. CrossRef Medline

Grillner S (2003) The motor infrastructure: from ion channels to neuronal networks. Nat Rev Neurosci 4:573-586. CrossRef Medline

Grillner S, Jessell TM (2009) Measured motion: searching for simplicity in spinal locomotor networks. Curr Opin Neurobiol 19:572-586. CrossRef Medline

Hägglund M, Borgius L, Dougherty KJ, Kiehn O (2010) Activation of groups of excitatory neurons in the mammalian spinal cord or hindbrain evokes locomotion. Nat Neurosci 13:246-252. CrossRef Medline

Hale ME, Ritter DA, Fetcho JR (2001) A confocal study of spinal interneurons in living larval zebrafish. J Comp Neurol 437:1-16. CrossRef Medline

Kimura Y, Okamura Y, Higashijima S (2006) alx, a zebrafish homolog of Chx10, marks ipsilateral descending excitatory interneurons that participate in the regulation of spinal locomotor circuits. J Neurosci 26:56845697. CrossRef Medline

Kimura Y, Satou C, Higashijima S (2008) V2a and V2b neurons are generated by the final divisions of pair-producing progenitors in the zebrafish spinal cord. Development 135:3001-3005. CrossRef Medline

Kimura Y, Satou C, Fujioka S, Shoji W, Umeda K, Ishizuka T, Yawo H, Higashijima S (2013) Hindbrain V2a Neurons in the Excitation of Spinal Locomotor Circuits during Zebrafish Swimming. Curr Biol 23:843849. CrossRef Medline

Li WC (2011) Generation of locomotion rhythms without inhibition in vertebrates: the search for pacemaker neurons. Integrative and comparative biology 51:879-889. CrossRef Medline

Li WC, Soffe SR, Wolf E, Roberts A (2006) Persistent responses to brief stimuli: feedback excitation among brainstem neurons. J Neurosci 26: 4026-4035. CrossRef Medline

Masino MA, Fetcho JR (2005) Fictive swimming motor patterns in wild type and mutant larval zebrafish. J Neurophysiol 93:3177-3188. CrossRef Medline

McLean DL, Masino MA, Koh IY, Lindquist WB, Fetcho JR (2008) Continuous shifts in the active set of spinal interneurons during changes in locomotor speed. Nat Neurosci 11:1419-1429. CrossRef Medline

Roberts A, Li WC, Soffe SR, Wolf E (2008) Origin of excitatory drive to a spinal locomotor network. Brain Res Rev 57:22-28. CrossRef Medline

Roberts A, Li WC, Soffe SR (2010) How neurons generate behavior in a hatchling amphibian tadpole: an outline. Front Behav Neurosci 4:16. CrossRef Medline

Schoonheim PJ, Arrenberg AB, Del Bene F, Baier H (2010) Optogenetic localization and genetic perturbation of saccade-generating neurons in zebrafish. J Neurosci 30:7111-7120. CrossRef Medline

Scott EK, Mason L, Arrenberg AB, Ziv L, Gosse NJ, Xiao T, Chi NC, Asakawa K, Kawakami K, Baier H (2007) Targeting neural circuitry in zebrafish using GAL4 enhancer trapping. Nat Methods 4:323-326. CrossRef Medline

Shepherd GM, Grillner S (2010) Handbook of brain microcircuits. New York: Oxford UP.

Sillar KT (2010) Development of spinal motor networks controlling axial movements. In: Oxford handbook of developmental behavioral neuroscience (Bumberh MS, Freeman JH, Robinson SR, eds), pp 240-253. Oxford: Oxford UP.

Wyart C, Del Bene F, Warp E, Scott EK, Trauner D, Baier H, Isacoff EY (2009) Optogenetic dissection of a behavioural module in the vertebrate spinal cord. Nature 461:407-410. CrossRef Medline 